\title{
BOUNDARY VALUE PROBLEMS FOR SECOND ORDER DIFFERENTIAL EQUATIONS IN CONVEX SUBSETS OF A BANACH SPACE
}

\author{
BY \\ KLAUS SCHMITT( ${ }^{1}$ ) AND PETER VOLKMANN
}

ABSTRACT. Let $E$ be a real Banach space, $C$ a closed, convex subset of $E$ and $f:[0,1] \times E \times E \rightarrow E$ be continuous. Let $u_{0}, u_{1} \in C$ and consider the boundary value problem

(*)

$$
u^{\prime \prime}=f\left(t, u, u^{\prime}\right), \quad u(0)=u_{0}, \quad u(1)=u_{1} .
$$

We establish sufficient conditions in order that $(*)$ have a solution $u: \mid 0,1] \rightarrow C$.

Introduction. Let $C$ be a closed, convex subset of the real Banach space $E$ and let $f:[0,1] \times C \times E \rightarrow E$ be a function with the property

$$
\left.\begin{array}{l}
\varphi \in E^{*}\left({ }^{2}\right), x \in C, \varphi(x)=\max _{q \in C} \varphi(q) \\
y \in E, \varphi(y)=0,0 \leqslant t \leqslant 1
\end{array}\right\} \Rightarrow \varphi(f(t, x, y)) \geqslant 0 .
$$

In this paper we show that under some additional (sometimes rather restrictive) assumptions the boundary value problem (BVP)

$$
u^{\prime \prime}=f\left(t, u, u^{\prime}\right), \quad u(0)=u_{0}, \quad u(1)=u_{1}, \quad 0 \leqslant t \leqslant 1,
$$

$\left(u_{0}, u_{1} \in C\right)$ has a solution $u:[0,1] \rightarrow C$. We note that (1) describes the behavior of $f$ on the boundary $\partial C$ of $C$, for if $\varphi \neq 0$, then condition (1) implies $x$ $\in \partial C$. In case $E=\mathrm{R}^{n}, n$-dimensional Euclidean space, and $C$ is bounded with int $C\left({ }^{3}\right) \neq \varnothing$, various results of this type exist in the literature (see e.g. [5] for a survey of such results). In this finite dimensional situation the general case may easily be obtained by projection methods. On the other hand, if $E$ is infinite dimensional, certain additional assumptions, either on $E$ or on $f$ seem to be needed to pass from the case int $C \neq \varnothing$ to the general case.

The paper is divided into two parts. In the first part we assume $f(t, x, y)$

Received by the editors August 23, 1974.

AMS (MOS) subject classifications (1970). Primary 34B15, $34 \mathrm{G05}$.

Key words and phrases. Boundary value problems, second order differential equations in Banach spaces.

(1) Research was performed while the first named author was a Visiting Professor at Universität Karlsruhe. His research was supported in part by U. S. Army research grant OAH-C-04-74-G-0208.

(2) $E^{*}$ denotes the space of all bounded linear functionals on $E$.

(3) "int" denotes the interior of a set. 
to be completely continuous and satisfy a Nagumo type growth condition with respect to $y$. Then it is known [6] that if $C$ is bounded and int $C \neq \varnothing$, the BVP (2) has a solution $u:[0,1] \rightarrow C$. In Theorem 1 we show that the same conclusion holds in case $C$ is a closed, bounded, convex subset of a uniformly convex space $E$, or in case $C$ is a compact convex subset. (The existence of a solution $u:[0,1] \rightarrow C$ of (2) for certain compact convex $C$ in $l^{p}, 1<p<\infty$, has already been treated by Thompson [7]; his methods, however, are quite different from ours.) In the second part we assume $f$ in (2) to be independent of $u^{\prime}$, continuous on $[0,1] \times E$ and satisfy a Lipschitz condition

$$
\|f(t, x)-f(t, y)\| \leqslant L\|x-y\|, \quad x, y \in E,
$$

where $L<\pi^{2}$. Under these assumptions the existence of a unique solution $u$ : $[0,1] \rightarrow E$ of $(2)$ follows easily by means of the contraction mapping principle, see e.g. [1] where the one dimensional case is treated, so one only needs to show that $u:[0,1] \rightarrow C$. This is done (Theorem 2 ) by using results and techniques formerly used by Redheffer and Walter [4] and in [8], [9], [10] in the study of invariance properties of sets relative to initial value problems for first order equations. A final result (Theorem 3 ) shows that it suffices to assume $f$ to be defined on $[0,1] \times C$, provided the continuity of $f$ relative to $t$ is uniform with respect to $x \in C$.

1. Completely continuous right-hand sides. Throughout this section we assume that $f:[0,1] \times C \times E \rightarrow E$ is completely continuous.

THEOREM 1. Let $C$ be a closed, bounded, convex subset of $E$ and assume there exists a continuous projection $P: E \rightarrow C$ assigning to each $x \in E$ a nearest point $P x \in C$ (i.e., $\|x-P x\|=\operatorname{dist}(C, x) \equiv \inf _{q \in C}\|q-x\|$; such $P$ always exists if the Banach space $E$ is uniformly convex in the sense of Clarkson [2]), or assume $C$ is compact. Let $u_{0}, u_{1} \in C$ and let $f$ satisfy (1) and the growth condition

$$
\|f(t, x, y)\| \leqslant \omega(\|y\|) \quad(0 \leqslant t \leqslant 1, x \in C, y \in E),
$$

where $\omega:[0, \infty) \rightarrow(0, \infty)$ is a continuous nondecreasing function with $\lim _{s \rightarrow \infty} s^{2} / \omega(s)=\infty$. Then the BVP (2) has a solution $u:[0,1] \rightarrow C$.

Proof. 1. If $C$ is closed, bounded, convex and int $C \neq \varnothing$, the above result holds without further assumptions on $C,[6$, Theorem 4.1].

2. A further result [6, Lemma 2.1] which is needed in what is to follow and which makes use of the properties of $\omega$ is the following: For each $R>$ 0 there exists $M$ (depending only on $R$ and $\omega$ ) such that: if $u:[0,1] \rightarrow E$ is twice continuously differentiable and 


$$
\|u(t)\| \leqslant R, \quad\left\|u^{\prime \prime}(t)\right\| \leqslant \omega\left(\left\|u^{\prime}(t)\right\|\right), \quad 0 \leqslant t \leqslant 1,
$$

then $\left\|u^{\prime}(t)\right\| \leqslant M, 0 \leqslant t \leqslant 1$.

3. Let $C$ be such that there exists a continuous projection $P: E \rightarrow C$ as in the statement of Theorem 1. Define $\widetilde{f}:[0,1] \times E \times E \rightarrow E$ by

$$
\tilde{f}(t, x, y)=f(t, P x, y) \text {. }
$$

For each $\epsilon>0$ the set $C_{\epsilon}$ defined by

$$
C_{\epsilon}=\{x \in E: \operatorname{dist}(C, x) \leqslant \epsilon\}
$$

is a closed, bounded, convex subset of $E$ with int $C_{\epsilon} \neq \varnothing$. We shall show next that the result of [6] stated in 1. above may be applied to $\widetilde{f}$ and $C_{\epsilon}$.

Obviously $\tilde{f}$ is completely continuous and verifies the estimate

$$
\|\widetilde{f}(t, x, y)\| \leqslant \omega(\|y\|) \quad(0 \leqslant t \leqslant 1, x, y \in E) .
$$

Let us show (1) with $C$ and $f$ replaced by $C_{\epsilon}$ and $\widetilde{f}$, respectively, i.e.

$$
\left.\begin{array}{l}
\varphi \in E^{*}, x \in C_{\epsilon}, \varphi(x)=\max _{q \in C_{\epsilon}} \varphi(q) \\
y \in E, \varphi(y)=0,0 \leqslant t \leqslant 1
\end{array}\right\} \Rightarrow \varphi(\tilde{f}(t, x, y)) \geqslant 0 .
$$

Let $x \in C_{\epsilon}$, then $\|x-P x\| \leqslant \epsilon$. Thus, if $q \in C$, we have that $q+x-P x \in C_{\epsilon}$. The hypotheses of $(6)$ consequently imply

$$
\varphi(x) \geqslant \varphi(q+x-P x)=\varphi(q)+\varphi(x)-\varphi(P x),
$$

and since $q \in C$ was arbitrary, it follows that

$$
\varphi(P x)=\max _{q \in C} \varphi(q)
$$

Using (1), we therefore obtain

$$
\varphi(\widetilde{f}(t, x, y))=\varphi(f(t, P x, y)) \geqslant 0,
$$

proving (6).

Using Theorem 4.1 of [6] we conclude the existence of a solution $u_{e}$ : $[0,1] \rightarrow C_{\epsilon}$ of the BVP

$$
u_{\epsilon}^{\prime \prime}=\tilde{f}\left(t, u_{\epsilon}, u_{\epsilon}^{\prime}\right), \quad u_{\epsilon}(0)=u_{0}, \quad u_{\epsilon}(1)=u_{1} .
$$

4. We now employ a limiting process (letting $\epsilon \rightarrow 0$ ) to obtain the desired conclusion.

Let $\left\{\epsilon_{n}\right\}$ be a monotone decreasing sequence of real numbers with $\lim _{n \rightarrow \infty} \epsilon_{n}=0$. Denote by $u_{n}=u_{\epsilon_{n}}$, where $u_{\epsilon_{n}}:[0,1] \rightarrow C_{\epsilon_{n}}$ is a solution of (7), with $\epsilon$ replaced by $\epsilon_{n}$. Choose $R>0$ such that $\left\|u_{n}(t)\right\| \leqslant R, 0 \leqslant t \leqslant 1, n$ $=1,2, \ldots$ Using (5) and 2. we obtain the existence of a constant $M>0$ such that $\left\|u_{n}^{\prime}(t)\right\| \leqslant M, 0 \leqslant t \leqslant 1, n=1,2, \ldots$.

Let $G$ denote the Green's function 


$$
G(t, s)= \begin{cases}-s(1-t), & 0 \leqslant s \leqslant t \leqslant 1 \\ -t(1-s), & 0 \leqslant t \leqslant s \leqslant 1\end{cases}
$$

then

$$
u_{n}(t)=\int_{0}^{1} G(t, s) \tilde{f}\left(s, u_{n}(s), u_{n}^{\prime}(s)\right) d s+(1-t) u_{0}+t u_{1}
$$

and

$$
u_{n}^{\prime}(t)=\int_{0}^{1} \frac{\partial}{\partial t} G(t, s) \widetilde{f}\left(s, u_{n}(s), u_{n}^{\prime}(s)\right) d s+u_{1}-u_{0}
$$

Using the complete continuity of $\widetilde{f}$, the uniform boundedness of $\left\{u_{n}\right\}$, $\left\{u_{n}^{\prime}\right\}$ and (8), (9) we conclude that $\left\{u_{n}\right\}$ and $\left\{u_{n}^{\prime}\right\}$ are equicontinuous sequences and that there exists a compact set $K \subseteq E$ such that $u_{n}(t), u_{n}^{\prime}(t) \in K, 0 \leqslant t \leqslant 1$, $n=1,2, \ldots$.

We may thus employ the theorem of Ascoli-Arzelà to obtain a subsequence of $\left\{u_{n}\right\}$ which converges to a solution $u$ of

$$
u^{\prime \prime}=\tilde{f}\left(t, u, u^{\prime}\right), \quad u(0)=u_{0}, \quad u(1)=u_{1}, \quad 0 \leqslant t \leqslant 1 .
$$

Since, further, $\operatorname{dist}\left(C, u_{n}(t)\right) \leqslant \epsilon_{n}$ and $\lim _{n \rightarrow \infty} \epsilon_{n}=0$, we obtain $\operatorname{dist}(C, u(t))=$ 0 , from which follows that $u:[0,1] \rightarrow C$ and $\widetilde{f}\left(t, u, u^{\prime}\right)=f\left(t, u, u^{\prime}\right)$, proving that $u$ is a solution of (2).

5. We next consider the case where $C$ is a compact convex subset of $E$ (here no additional assumptions on $E$ are needed). Choose $R>0$ such that: $x \in$ $C \Rightarrow\|x\| \leqslant R$. Determine $M=M(R, \omega)$ according to 2. above. Define $Q: E \rightarrow$ $E$ by

$$
Q y= \begin{cases}y, & \|y\| \leqslant M, \\ M y /\|y\|, & \|y\|>M,\end{cases}
$$

and put

$$
\widetilde{f}(t, x, y)=f(t, x, Q y) \quad(0 \leqslant t \leqslant 1, x \in C, y \in E) .
$$

The complete continuity of $f$ implies that of $\widetilde{f}$. Hence the range of $\widetilde{f}$ is contained in some compact set $K \subseteq E$, and (1) and (4) are satisfied by $\widetilde{f}$.

Let $E_{1}$ denote the closed linear span of $C, K$ and restrict $\widetilde{f}$ to $\widetilde{f}:[0,1] \times$ $C \times E_{1} \rightarrow E_{1}$. Since $C$ and $K$ are compact, $E_{1}$ is a separable Banach space. Using a result of Clarkson [2] we may equip $E_{1}$ with a new norm $\|\cdot\|_{1}$, equivalent to $\|\cdot\|$, such that $E_{1}$ becomes strictly convex. Hence to each $x \in E_{1}$ there corresponds a unique nearest point (with respect to $\|\cdot\|_{1}$ ) $P x$ in $C$. Since (1) holds with $E, f$ replaced by $E_{1}, \widetilde{f}\left(\varphi \in E_{1}^{*}\right.$ with $\varphi(x)=\max _{q \in C} \varphi(q)$ is extendable to a $\Phi \in E^{*}$ with the same property) and since $\widetilde{f}$ is bounded and the projection $P$, just defined, is continuous, we may apply the arguments of 3 . and 4 . to obtain a 
solution $u:[0,1] \rightarrow C$ of

$$
u^{\prime \prime}=\tilde{f}\left(t, u, u^{\prime}\right), \quad u(0)=u_{0}, \quad u(1)=u_{1}, \quad 0 \leqslant t \leqslant 1 .
$$

Returning to the original norm we have that $\|u(t)\| \leqslant R, 0 \leqslant t \leqslant 1$, and by the monotonicity of $\omega$ we find $\left\|u^{\prime \prime}(t)\right\| \leqslant \omega\left(\left\|u^{\prime}(t)\right\|\right)$, implying $\left\|u^{\prime}(t)\right\| \leqslant M, 0 \leqslant t \leqslant$ 1. Hence the definition of $\widetilde{f}$ shows that $u$ is a solution of (2).

2. Right-hand sides satisfying a Lipschitz condition. Throughout this section we shall assume that $f$ is independent of $u^{\prime}$ and satisfies a Lipschitz condition

$$
\|f(t, x)-f(t, y)\| \leqslant L\|x-y\| \quad(0 \leqslant t \leqslant 1 ; x, y \in E) .
$$

Theorem 2. Let $C$ be a closed, convex subset of $E$ and let $u_{0}, u_{1} \in C$. Assume that $f:[0,1] \times E \rightarrow E$ is continuous and satisfies the Lipschitz condition (11) with $L<\pi^{2}$. Further assume

$$
\left(0 \leqslant t \leqslant 1, \varphi \in E^{*}, x \in C, \varphi(x)=\max _{q \in C} \varphi(q)\right) \Rightarrow \varphi(f(t, x)) \geqslant 0 .
$$

Then the BVP

$$
u^{\prime \prime}=f(t, u), \quad u(0)=u_{0}, \quad u(1)=u_{1}, \quad 0 \leqslant t \leqslant 1,
$$

has a unique solution $u:[0,1] \rightarrow C$.

Proof. 1. For our proof we need a formula first established for closed, convex cones by Redheffer and Walter [4] equivalent to (12):

$$
\lim _{h \rightarrow 0+} \frac{1}{h} \operatorname{dist}(C, x-h f(t, x))=0 \quad(0 \leqslant t \leqslant 1, x \in C)
$$

(see [8]). Letting (for $\xi \geqslant 0$ )

$$
C_{\xi}=\{x \in E: \operatorname{dist}(C, x) \leqslant \xi\}
$$

$\left(C_{0}=C\right)$, using (11) and (12) and a result from [10] we obtain

$$
\lim _{h \rightarrow 0+} \frac{1}{h} \operatorname{dist}\left(C_{\xi}, x-h f(t, x)\right) \leqslant L \xi \quad\left(0 \leqslant t \leqslant 1, x \in C_{\xi}\right) .
$$

(In [10] this formula is written with lim sup in place of lim, however, since $C$ is convex the limit exists.)

2. Let $\widetilde{E}=E \oplus \mathbf{R}$ normed by $\|(x, \xi)\|=\max (\|x\|,|\xi|)$. With $p=(\theta, 1)$ $(\theta=$ zero element of $E)$ we may write

$$
\widetilde{E}=E \oplus \mathbf{R}=\{x+\xi p: x \in E, \xi \in \mathbf{R}\} .
$$

Via the natural embedding, we consider $E$ as a subspace of $\widetilde{E}$. Let

$$
\widetilde{C}=\{x+\xi p: \operatorname{dist}(C, x) \leqslant \xi\},
$$

then $\widetilde{C}$ is a closed, convex subset of $\widetilde{E}$ with nonempty interior. Define $\widetilde{f}:[0,1]$ 
$\times \widetilde{C} \rightarrow \widetilde{E}$ by

$$
\widetilde{f}(t, x+\xi p)=f(t, x)-L \xi p \quad(0 \leqslant t \leqslant 1, x+\xi p \in \widetilde{C}) .
$$

Then $\widetilde{f}$ is continuous and satisfies a Lipschitz condition with Lipschitz constant $L$ with respect to its second argument:

$$
\|\tilde{f}(t, \tilde{x})-\tilde{f}(t, \tilde{y})\| \leqslant L\|\tilde{x}-\tilde{y}\| \quad(0 \leqslant t \leqslant 1, \tilde{x}, \tilde{y} \in \tilde{C}) .
$$

Our method of proof requires a condition analogous to (12) for $\widetilde{f}$ and $\widetilde{C}$, namely:

$$
\left(0 \leqslant t \leqslant 1, \tilde{\varphi} \in \widetilde{E}^{*}, \tilde{x} \in \tilde{C}, \tilde{\varphi}(\tilde{x})=\max _{\tilde{q} \in \tilde{C}} \tilde{\varphi}(\tilde{q})\right) \Rightarrow \tilde{\varphi}(\tilde{f}(t, \tilde{x})) \geqslant 0 .
$$

That (18) follows from (12) has already been sketched in [9] for the case where $C$ is a closed, convex cone; our proof to follow is patterned after the one in [9]. (For general closed, convex $C(18)$ has been established in [8] for $f$ defined by $\widetilde{f}(t, x+\xi p)=f(t, x)-4 L \xi p$. That result, however, is not sufficient for our purposes.)

3. To prove (18) we use the equivalence of (12) and (14) (applied to $\widetilde{C}$ and $\widetilde{f}$ ) and verify

$$
\lim _{h \rightarrow 0+} \frac{1}{h} \operatorname{dist}(\widetilde{C}, \tilde{x}-h \widetilde{f}(t, \tilde{x}))=0 \quad(0 \leqslant t \leqslant 1, \tilde{x} \in \widetilde{C}) .
$$

Let $t \in[0,1]$ and. $\tilde{x}=x+\xi p \in \widetilde{C}$, i.e., $x \in C_{\xi}$. Then (15) implies that for $\epsilon>$ 0 there exists $h_{0}(\epsilon)$ such that

$$
h^{-1} \operatorname{dist}\left(C_{\xi}, x-h f(t, x)\right)<L \xi+\epsilon \quad\left(0<h \leqslant h_{0}(\epsilon)\right) .
$$

Thus there exists $y_{h} \in C_{\xi}$ (i.e. $y_{h}+\xi p \in \widetilde{C}$ ) such that

$$
\left\|x-h f(t, x)-y_{h}\right\|<h L \xi+h \epsilon,
$$

implying

$$
x-h f(t, x)-y_{h}+h(L \xi+\epsilon) p \in \widetilde{K} \equiv\{y+\eta p: y \in E,\|y\| \leqslant \eta\} .
$$

Now $\widetilde{C}+\widetilde{K} \subseteq \widetilde{C}$ and $y_{h}+\xi p \in \widetilde{C}$, yielding

$$
x+\xi p-h[f(t, x)-L \xi p]+h \in p \in \widetilde{C},
$$

from which, in turn, it follows that

$$
h^{-1} \operatorname{dist}(\tilde{C}, \tilde{x}-h \tilde{f}(t, \tilde{x})) \leqslant \epsilon \quad\left(0<h \leqslant h_{0}(\epsilon)\right)
$$

implying (19).

4. Define $P: \widetilde{E} \rightarrow \widetilde{C}$ by

$$
P(x+\xi p)= \begin{cases}x+\xi p, & \operatorname{dist}(C, x) \leqslant \xi, \\ x+\operatorname{dist}(C, x) p, & \operatorname{dist}(C, x)>\xi .\end{cases}
$$

Then it is easily seen that 


$$
\|P(\tilde{x})-P(\tilde{y})\| \leqslant\|\tilde{x}-\tilde{y}\| .
$$

Extending $\widetilde{f}$ to $[0,1] \times \widetilde{E}$ by setting

$$
\tilde{f}(t, \tilde{x})=\tilde{f}(t, P \tilde{x}) \quad(0 \leqslant t \leqslant 1, \tilde{x} \in \widetilde{E}),
$$

we see by (21) that (17) remains valid for the extended function (with the same Lipschitz constant).

Letting

$$
\widetilde{C}_{\eta}=\widetilde{C}-\eta p=\{\tilde{x}-\eta p: \tilde{x} \in \widetilde{C}\} \quad\left(\eta \geqslant 0 ; \widetilde{C}_{0}=\widetilde{C}\right)
$$

we see that (18) holds with $\widetilde{C}$ replaced by $\widetilde{C}_{\eta}$, i.e.,

(23) $\left(0 \leqslant t \leqslant 1, \tilde{\varphi} \in \widetilde{E}^{*}, \tilde{x} \in \widetilde{C}_{\eta}, \tilde{\varphi}(\tilde{x})=\max _{\tilde{q} \in \widetilde{C}_{\eta}} \tilde{\varphi}(\tilde{q})\right) \Rightarrow \tilde{\varphi}(\tilde{f}(t, \tilde{x})) \geqslant 0$,

for if $\tilde{x}=x+\xi p$ and $\tilde{\varphi} \neq 0$ satisfy the hypotheses of (23), then $\tilde{x} \in \partial \widetilde{C}_{\eta}$ and therefore $x+(\xi+\eta) p=\tilde{x}+\eta p \in \partial \widetilde{C}$. Thus $\operatorname{dist}(C, x)=\xi+\eta$, which combined with (20) yields $P \tilde{x}=x+(\xi+\eta) p=\tilde{x}+\eta p$. Therefore $\tilde{\varphi}(\tilde{P \tilde{x}})=$

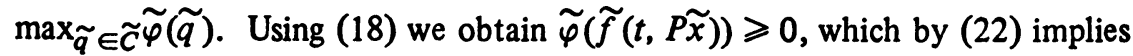
(23).

5. The function $\sigma: \widetilde{E} \rightarrow \mathbf{R}$, defined by

$$
\sigma(x+\xi p)= \begin{cases}0, & \operatorname{dist}(C, x) \leqslant \xi, \\ \operatorname{dist}(C, x)-\xi, & \operatorname{dist}(C, x)>\xi,\end{cases}
$$

satisfies a Lipschitz condition with Lipschitz constant 2. Choose $\epsilon>0$ such that $L_{1}=L+2 \epsilon<\pi^{2}$. Then

$$
\hat{f}(t, \tilde{x})=\tilde{f}(t, \tilde{x})-\epsilon \sigma(\tilde{x}) p
$$

satisfies

$$
\|\hat{f}(t, \tilde{x})-\hat{f}(t, \tilde{y})\| \leqslant L_{1}\|\tilde{x}-\tilde{y}\| \quad(0 \leqslant t \leqslant 1, \tilde{x}, \tilde{y} \in \widetilde{E})
$$

further it follows from (23) and (24) that

$$
\begin{gathered}
\left(0 \leqslant t \leqslant 1, \eta>0, \tilde{\varphi} \in \widetilde{E}^{*}, \tilde{\varphi} \neq 0, \tilde{x} \in \widetilde{C}_{\eta}, \tilde{\varphi}(\tilde{x})=\max _{\tilde{q} \in \widetilde{C}_{\eta}} \tilde{\varphi}(\tilde{q})\right) \\
\Rightarrow \tilde{\varphi}(\hat{f}(t, \tilde{x}))>0 .
\end{gathered}
$$

Because $L_{1}<\pi^{2}$, the BVP

$$
\tilde{u}^{\prime \prime}=\hat{f}(t, \tilde{u}), \quad \tilde{u}(0)=u_{0}, \quad \tilde{u}(1)=u_{1},
$$

has a unique solution $\tilde{u}:[0,1] \rightarrow \widetilde{E}$ (this fact has already been mentioned in the introduction). It is the purpose of the next paragraphs to show that $\tilde{u}$ is a solution of (13) with values in $C$. 
6. There exists a smallest $\eta \geqslant 0$ such that $\tilde{u}:[0,1] \rightarrow \widetilde{C}_{\eta}(\tilde{u}$ is the solution of (26)). Suppose $\eta>0$. Then there exists $t_{0} \in(0,1)$ such that $\tilde{u}\left(t_{0}\right) \in$ $\partial \widetilde{C}_{\eta}\left(\tilde{u}(0), \tilde{u}(1) \in\right.$ int $\left.\widetilde{C}_{\eta}\right)$. We may thus choose $\tilde{\varphi} \in \widetilde{E}^{*}, \tilde{\varphi} \neq 0$, such that $\tilde{\varphi}\left(\tilde{u}\left(t_{0}\right)\right)=\max _{\tilde{q} \in \tilde{C}_{\eta}} \tilde{\varphi}(\tilde{q})$. By (25)

$$
\tilde{\varphi}\left(\hat{f}\left(t_{0}, \tilde{u}\left(t_{0}\right)\right)\right)>0 .
$$

On the other hand, the scalar function $\rho(t)=\tilde{\varphi}(\tilde{u}(t)), 0 \leqslant t \leqslant 1$, attains its maximum at $t_{0}$, hence $\rho^{\prime \prime}\left(t_{0}\right) \leqslant 0$. But

$$
\rho^{\prime \prime}\left(t_{0}\right)=\tilde{\varphi}\left(\tilde{u}^{\prime \prime}\left(t_{0}\right)\right)=\tilde{\varphi}\left(\hat{f}\left(t_{0}, \tilde{u}\left(t_{0}\right)\right)\right),
$$

contradicting (27). Thus $\tilde{u}:[0,1] \rightarrow \widetilde{C}_{0}=\widetilde{C}$.

7. It now follows from the definition of $\hat{f}$ that $\hat{f}(t, \tilde{u}(t))=\tilde{f}(t, \tilde{u}(t))$. Thus $\tilde{u}$ is the solution of the BVP

$$
\tilde{u}^{\prime \prime}=\tilde{f}(t, \tilde{u}), \tilde{u}(0)=u_{0}, \quad \tilde{u}(1)=u_{1} .
$$

Using the notation

$$
\tilde{u}(t)=u(t)+\xi(t) p \quad(u(t) \in E, \xi(t) \in \mathbf{R}, 0 \leqslant t \leqslant 1),
$$

we may decompose (28) into

$$
\begin{array}{lll}
u^{\prime \prime}=f(t, u), & u(0)=u_{0}, & u(1)=u_{1}, \\
\xi^{\prime \prime}=-L \xi, & \xi(0)=0, \quad \xi(1)=0,
\end{array}
$$

with the further constraint

$$
\operatorname{dist}(C, u(t)) \leqslant \xi(t) .
$$

Since, however, $L<\pi^{2}$, it follows that $\xi(t) \equiv 0$, and thus $\operatorname{dist}(C, u(t))=0$, i.e., $u:[0,1] \rightarrow C$. This completes the proof of Theorem 2 .

THEOREM 3. Theorem 2 remains valid if $f(t, x)$ is only defined on $[0,1]$ $x C$, but is uniformly continuous in $t$ with respect to $x$, i.e.,

$$
\sup _{x \in C}\left\|f\left(t_{n}, x\right)-f(t, x)\right\| \rightarrow 0 \text { as } t_{n} \rightarrow t .
$$

Proof. We embed $E$ via an isometric isomorphism in some Banach space $B(S)$ of bounded functions on some set $S$ (e.g. $S=\left\{\varphi \in E^{*}:\|\varphi\| \leqslant 1\right\}$ ). Then (12) remains valid with $B(S)^{*}$ in place of $E^{*}$. Thus we may consider the problem in $B(S)$ instead of $E$; in particular we may consider $f:[0,1] \times C \rightarrow B(S)$, where $C \subseteq B(S)$. By adopting the coordinate conventions and writing the elements $z \in$ $B(S)$ as $z=\left(z_{\sigma}\right)_{\sigma \in S}\left(z_{\sigma} \in R,\|z\|=\sup _{\sigma \in S}\left|z_{\sigma}\right|\right)$, we define $f_{\sigma}:[0,1] \times C \rightarrow$ $\mathbf{R}(\sigma \in S)$ by

$$
f_{\sigma}(t, x)=f(t, x)_{\sigma} \quad(0 \leqslant t \leqslant 1, x \in C, \sigma \in S)
$$


The Lipschitz continuity of $f$ implies that of $f_{\sigma}$, i.e.,

$$
\left|f_{\sigma}(t, x)-f_{\sigma}(t, y)\right| \leqslant L\|x-y\| \quad(0 \leqslant t \leqslant 1, x, y \in C, \sigma \in S) .
$$

A result of McShane [3] implies that the function

$$
\tilde{f}_{\sigma}(t, x)=\sup _{q \in C}\left(f_{\sigma}(t, q)-L\|q-x\|\right) \quad(x \in B(S))
$$

is an extension of $f_{\sigma}$ to $[0,1] \times B(S)$, such that

$$
\left|\tilde{f}_{\sigma}(t, x)-\tilde{f}_{\sigma}(t, y)\right| \leqslant L\|x-y\| \quad(0 \leqslant t \leqslant 1, x, y \in B(S), \sigma \in S) .
$$

Define $\widetilde{f}:[0,1] \times B(S) \rightarrow B(S)$ by

$$
\widetilde{f}(t, x)_{\sigma}=\widetilde{f_{\sigma}}(t, x) \quad(0 \leqslant t \leqslant 1, x \in B(S), \sigma \in S) .
$$

Then $\tilde{f}$ is an extension of $f$ to $[0,1] \times B(S)$ and satisfies (11). By $(32) \tilde{f}(t, x)$ is also continuous with respect to $t$. We may therefore use Theorem 2 to conclude that the BVP

$$
u^{\prime \prime}=\tilde{f}(t, u), \quad u(0)=u_{0}, \quad u(1)=u_{1} \quad\left(u_{0}, u_{1} \in C\right)
$$

has a solution $u:[0,1] \rightarrow C$. Since $\widetilde{f}$ is an extension of $f, u$ is a solution of the original problem.

\section{REFERENCES}

1. P. B. Bailey, L. F. Shampine and P. E. Waltman, Nonlinear two point boundary value problems, Math. in Sci. and Engineering, vol. 44, Academic Press, New York and London, 1968. MR 37 \#6524. 396-414.

2. J. A. Clarkson, Uniformly convex spaces, Trans. Amer. Math. Soc. 40 (1936), $837-842$.

3. E. J. McShane, Extension of range of functions, Bull. Amer. Math. Soc. 40 (1934),

4. R. M. Redheffer and W. Walter, Flow-invariant sets and differential inequalities in normed spaces, Applicable Anal. (to appear).

5. K. Schmitt, Randwertaufgaben für gewöhnliche Differentialgleichungen, Proc. Steiermark. Math. Symposium, VII, Graz, Austria, 1973, pp. 1-55.

6. K. Schmitt and R. C. Thompson, Boundary value problems for infinite systems of second-order differential equations, J. Differential Equations 18 (1975), 277-295.

7. R. C. Thompson, Differential inequalities for infinite second order systems and an application to the method of lines, J. Differential Equations 17 (1975), $421-434$.

8. P. Volkmann, Über die Invarianz konvexer Mengen und Differentialungleichungen in einem normierten Raume, Math. Ann. 203 (1973), 201-210. MR 48 \#667.

9. - Gewöhnliche Differentialungleichungen mit quasimonoton wachsenden Funktionen in Banachräumen (Proc. Conf. Ordinary Partial Differential Equations, Dundee, 1974), Lecture Notes in Math., vol. 415, Springer-Verlag, Berlin and New York, 1974, pp. 439-443.

10. - Über die positive Invarianz einer abgeschlossenen Teilmenge eines Banachschen Raumes bezüglich der Differentialgleichung $u^{\prime}=f(t, u)$, J. Reine Angew. Math. (to appear).

\section{UTAH 84112}

DEPARTMENT OF MATHEMATICS, UNIVERSITY OF UTAH, SALT LAKE CITY,

MATHEMATISCHES INSTITUT I, UNIVERSITÄT KARLSRUHE (TH), 75 KARLSRUHE 1, FEDERAL REPUBLIC OF GERMANY 